\title{
Research on Comprehensive Evaluation of Freeway Traffic Safety in Heilongjiang Province
}

\author{
Jia LIU ${ }^{1, a,{ }^{*}}$, Weiwei YAN ${ }^{2, b}$ \\ ${ }^{1}$ Department of Management Engineering, Engineering University of PAP, Xi'an 710086, China \\ ${ }^{2}$ School of Economic and Management, Harbin Institute of Technology, Harbin 150086, China \\ agege20010228@163.com, b610038606@qq.com, *corresponding author
}

Keywords: Heilongjiang Province; Freeway; Safety; TOPSIS

\begin{abstract}
Through the statistic analysis of the accident data of Heilongjiang freeway from 2010 to 2014, it is concluded the regularities of distribution of the accidents on the Heilongjiang freeway, as well as the factors influencing the security of Heilongjiang freeway system. Then, the evaluation index of Heilongjiang freeway safety is built up. In order to intuitively compare the evaluation objects, the evaluation model of Heilongjiang freeway safety based on the method of Technique for Order Preference by Similarity to Ideal Solution (TOPSIS) is constructed. Finally, 7 national freeways in Heilongjiang province are selected as the evaluation objects, which are evaluated making use of this model.
\end{abstract}

\section{Introduction}

Freeway is characterized by high linear, well road conditions, closed all the way, and not pedestrians or low-speed traffic interference, so the condition of freeway traffic should be better than the ordinary road theoretically. According to the data, freeway traffic accident rate is much lower than the ordinary road in the developed countries, probably only about $1 / 10$ of the ordinary road traffic accident rate, and less fatality rate than ordinary road, about $1 / 3$ of the ordinary road accident fatality rate. However, it's just the opposite in China that the freeway accident rate and fatality rate are both higher than ordinary road.

Analyzing and evaluating the freeway safety and finding weaknesses are beneficial to grasp the safety level of freeway, control the situation broadly, so as to change the passive situation after the traffic accident.

More Western countries pay close attention to the management of freeway safety. The UK is one of the countries which earlier proposed road safety evaluation. In 1991 the IHT of UK evaluated the safety of roads nationwide, and wrote the "Guidelines to Road Safety Audit", which was the detailed guidance note for freeway safety evaluation [1]. Australia is another country taking much count of road safety early, and the theory and practice about road safety evaluation are more mature. In 1994, the AUSTROAD established a special organization to formulate guidelines evaluating freeway Safety, and published "Road Safety Audit" [2].

In the respect of comprehensive evaluation of freeway safety, methods adopted include the analytic hierarchy process [3], fuzzy comprehensive evaluation [4], grey evaluation, neural network [5], etc. Most researches were evaluating the safety of one freeway, and results were levels of "safe", "general safe”, etc., which were set subjectively. Such evaluation results of no reference could not intuitively descript the safety of freeway, could not propose the targeted improvement suggestion either.

\section{Analysis of Influence Factors of Heilongjiang Province Freeway System}

Through the statistical data, it is found that "driver-vehicle-freeway-environment" constitutes an organic whole, which driver is direct and main factor leading to traffic accident, but driver is the most subjective, the most difficult to change, and uncontrollable factor. So, in this organic whole of "driver-vehicle-freeway -environment", in addition to the driver $(D)$, the factors of vehicle $(V)$, 
freeway $(F)$ and environment $(E)$ also should be regard as key points, so as to analyze the cause of traffic accidents generally. As a result, the freeway safety $(S)$ is expressed as the following formula:

$$
S=f(D, V, F, E)
$$

On the basis of statistics of the freeway traffic accident in Heilongjiang province in 2010-2014, the percentage of accident numbers caused by each subsystem is shown in figure 1.

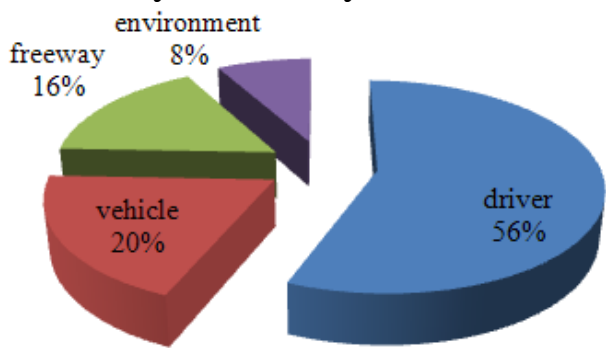

Fig.1 Impact on traffic accidents of each subsystem of Heilongjiang freeway

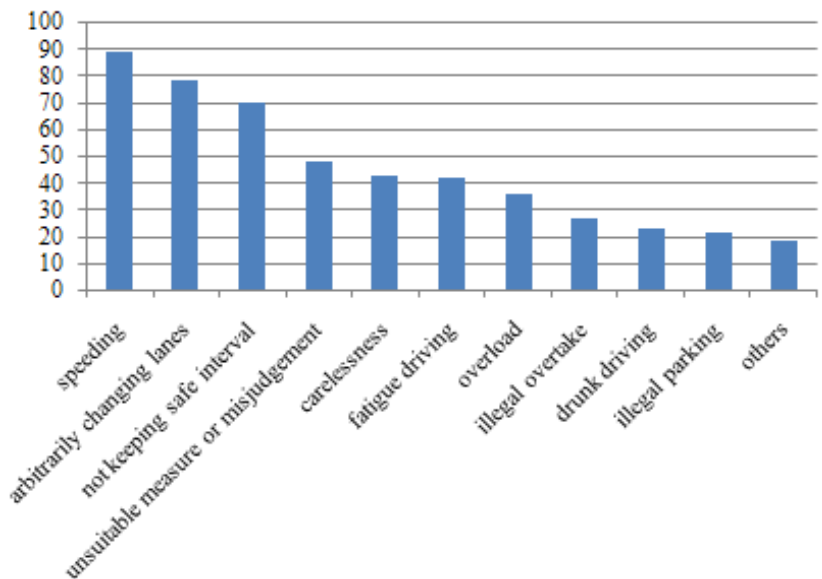

Fig.2 Various unlawful acts of Heilongjiang province freeway

Factor of Driver. All over the world, driver is main factor causing traffic accident. Heilongjiang freeway as well, the factor of driver accounts for $56 \%$. Through further statistics of various unlawful acts of 2010-2014 in Heilongjiang province, speeding, arbitrarily changing lanes, and not keeping safe interval are leading causes of traffic accident in Heilongjiang province freeway, as shown in figure 2.

Factor of Vehicle. Of the accidents caused by vehicle on Heilongjiang province freeways, the main fault is flat tire, the second is bad brake and brake failure. These three reasons account for $66 \%$ of total number of all the accidents caused by vehicle, as shown in figure 3 .

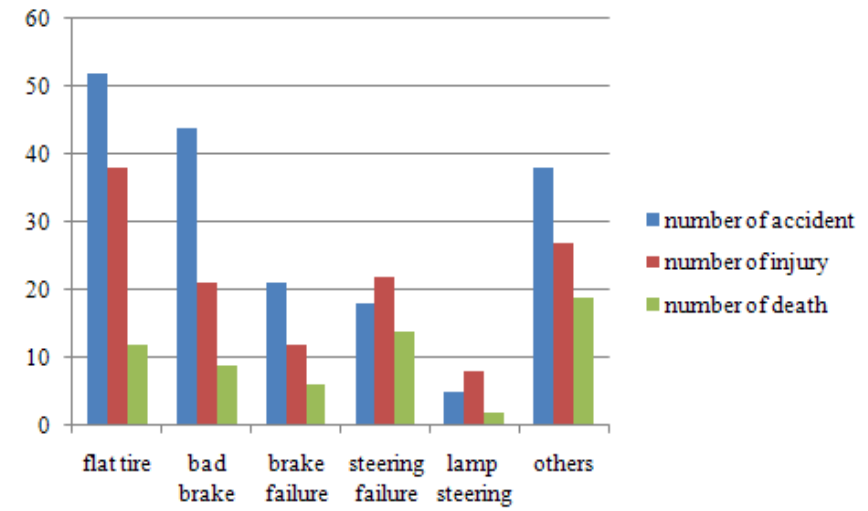

Fig.3 Statistic of accident caused by vehicle on Heilongjiang province freeway 


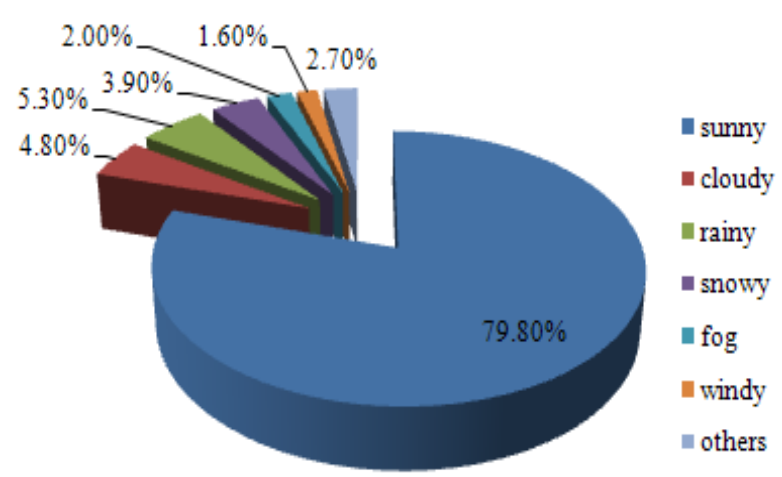

Fig.4 Statistics of traffic caused by bad weathers

Factor of Freeway. Freeway could trigger traffic accidents, and as part of factors. Factors of freeway affecting the traffic safety are road alignment, road conditions, safety facilities, etc.

Road alignments of freeway include horizontal alignment, profile alignment and the combination of both. In the process of driving vehicle will be affected by the road alignment. If the curvature of the horizontal alignment is bigger, the road bend degree is higher, and the corresponding accident rate will be higher. But it doesn't mean that accident rate of straight road will be less, because the driver driving in a straight line for long time will feel dull and tired. In addition, driving on the straight freeway usually doesn't have to brake, so the driver could speed unconsciously. These impacts on driver from straight road are easy to result in accidents. If the vertical curve radius of freeway is too small, the driver will not be able to observe the road ahead, cannot find abnormal situation, and cannot take measures in time. Freeway accident statistics of German show that when the slope is more than $4 \%$, the accident rate is greatly increased.

Road conditions (including the flatness and the sliding resistance) can also affect driving on the freeway. When speeding fast, even if a tiny damage, major accident could happen. When it rains, if the road cannot resist sliding well, there will produce "water film" which will reduce the friction between road surface and tires, and sliding resistance is insufficient. If drivers break under this condition, sideslip will be happened.

There are facilities such as traffic signs, marking, and fence etc., which are critical to reduce traffic accidents on freeway. These facilities improve the road landscape, provide drivers with vision induction, caution the front dangerous sections, etc., which play an important role to reduce the number of traffic accidents and relieve the severity.

Factor of Environment. Road environment generally includes bad weather and traffic flow, both affect drivers by means of influencing the driving conditions, which impact on traffic safety.

Factor of Weather. The weather is one of the important factors. The survey suggests that traffic accidents caused by adverse weather accounts for about $4.8 \%$ of the total number of traffic accident on Heilongjiang provincial freeway [6]. Among all the bad weather, rain and snow is the second factor. The proportion of accidents result from rain and snow is as high as $8 \%$, as shown in figure 4 .

Traffic Flow. In recent years, China's freeway mileage increases continuously, and traffic flow increases rapidly, so emergencies are more likely to be happened, and the congestion phenomenon occurs sometimes. Traffic flow creasing leads to the interval between vehicles is reduced, which makes drivers too nervous to take the correct and proper operation when emergency happens. Therefore, traffic flow affects the accident rate of freeway inevitably.

\section{Comprehensive Evaluation of Heilongjiang Freeway Traffic Safety}

Evaluation Index of Heilongjiang Freeway Traffic Safety.Based on the analysis of distribution regularity of Heilongjiang freeway traffic accidents, evaluation index of Heilongjiang freeway traffic safety is selected, as shown in figure 5 . 


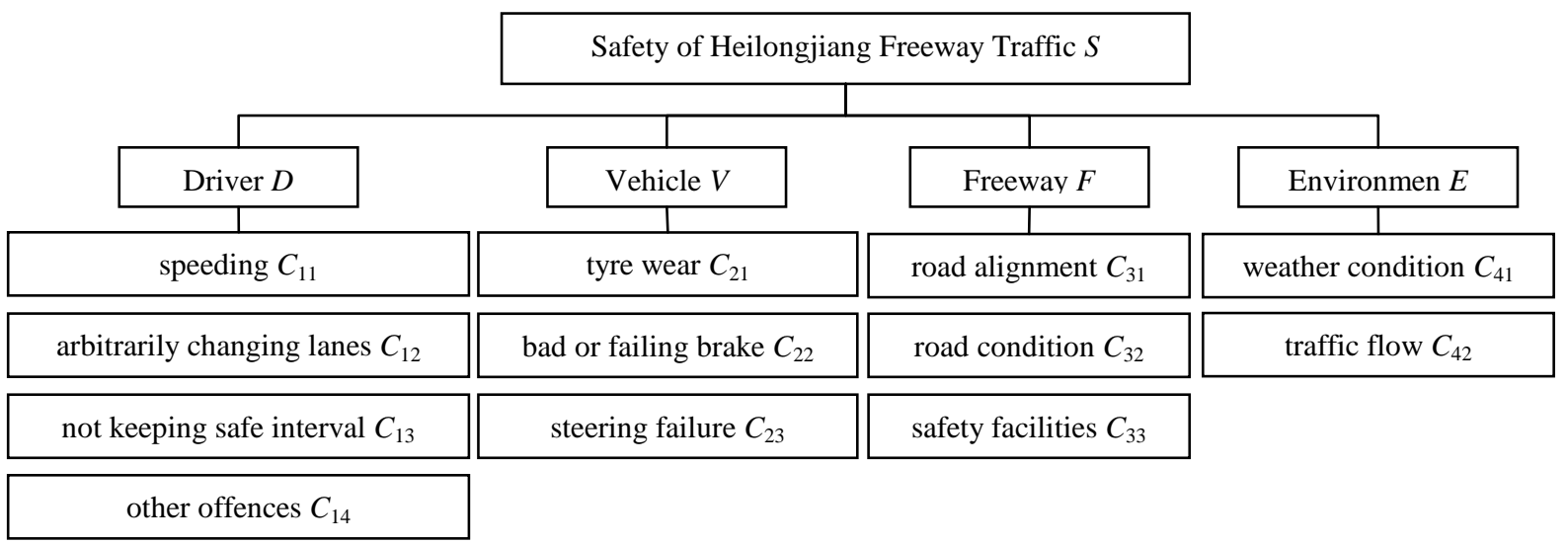

Fig.5 Evaluation index of Heilongjiang freeway traffic safety

Evaluation of Heilongjiang Freeway Traffic Safety Based on TOPSIS. 7 freeways of national level in Heilongjiang province are evaluated. By looking up "Notification of road accident Heilongjiang province”, and consulting relevant departments such as Heilongjiang traffic management bureau, most of indicators were collected from 2010 to 2014. Thereinto, road alignments $C_{31}$, road conditions $C_{32}$ and traffic facilities $C_{33}$ are not easy to be quantified, so obtain these data through questionnaires of 5 experts from relevant fields. Evaluation grade is divided into safer, safe, ordinarily safe, less safe and not safe, which are assigned 1, 3, 5, 7, 9 respectively. Expert evaluation is shown in Table 1.

Table 1 Expert evaluation of partial indicators

\begin{tabular}{clrrrrrc}
\hline \multirow{2}{*}{ Freeway } & \multirow{2}{*}{ Indicator } & \multicolumn{3}{c}{ Evaluation of 5 experts } & Mean \\
& & $P_{1}$ & $P_{2}$ & $P_{3}$ & $P_{4}$ & $P_{5}$ & value \\
\hline \multirow{2}{*}{ Beijing-Harbin } & road alignments $C_{31}$ & 5 & 3 & 3 & 3 & 1 & 3 \\
Freeway G1 & road conditions $C_{32}$ & 3 & 5 & 5 & 5 & 3 & 4.2 \\
& traffic facilities $C_{33}$ & 3 & 5 & 5 & 5 & 5 & 4.6 \\
\hline \multirow{2}{*}{ Hegang-Dalian } & road alignments $C_{31}$ & 1 & 3 & 1 & 3 & 1 & 1.8 \\
Freeway G11 & road conditions $C_{32}$ & 1 & 1 & 3 & 1 & 1 & 1.4 \\
& traffic facilities $C_{33}$ & 3 & 1 & 3 & 3 & 1 & 2.2 \\
\hline \multirow{2}{*}{ Hegang-Harbin } & road alignments $C_{31}$ & 3 & 5 & 1 & 3 & 3 & 3 \\
Freeway G1111 & road conditions $C_{32}$ & 3 & 1 & 5 & 3 & 3 & 3 \\
& traffic facilities $C_{33}$ & 1 & 3 & 3 & 3 & 3 & 2.6 \\
\hline \multirow{2}{*}{ Jilin-Heihe } & road alignments $C_{31}$ & 7 & 7 & 5 & 5 & 3 & 5.4 \\
Freeway G1211 & road conditions $C_{32}$ & 7 & 7 & 7 & 7 & 5 & 6.6 \\
& traffic facilities $C_{33}$ & 5 & 7 & 7 & 5 & 9 & 6 \\
\hline \multirow{2}{*}{ Harbin-Tongjiang } & road alignments $C_{31}$ & 7 & 5 & 5 & 7 & 7 & 6.2 \\
Freeway G1011 & road conditions $C_{32}$ & 7 & 7 & 5 & 5 & 5 & 5.8 \\
& traffic facilities $C_{33}$ & 7 & 7 & 7 & 7 & 9 & 7.2 \\
\hline \multirow{2}{*}{ Suifenhe-Manzhouli } & road alignments $C_{31}$ & 9 & 7 & 7 & 5 & 5 & 6.6 \\
Freeway G10 & road conditions $C_{32}$ & 5 & 5 & 5 & 7 & 3 & 5 \\
& traffic facilities $C_{33}$ & 5 & 5 & 3 & 5 & 7 & 5 \\
\hline \multirow{2}{*}{ Daqing-Guangzhou } & road alignments $C_{31}$ & 5 & 1 & 3 & 5 & 3 & 3.4 \\
Freeway G45 & road conditions $C_{32}$ & 3 & 3 & 3 & 3 & 1 & 2.6 \\
& traffic facilities $C_{33}$ & 3 & 1 & 5 & 3 & 5 & 3.2 \\
\hline
\end{tabular}

Evaluation of Heilongjiang Freeway Traffic Safety Based on TOPSIS. Combining the characteristics of system of freeway, 7 freeways are evaluated and ranked using the method of TOPSIS.

Suppose that there are $n$ evaluation objects $R=\left(R_{1}, R_{2}, \cdots, R_{n}\right), m$ evaluation indicators $C=\left(C_{1}, C_{2}, \cdots, C_{m}\right)$. The indicator $C_{i}$ of valuation object $R_{j}$ is $y_{i j}^{\prime}(i=1,2, \cdots, n ; j=1,2, \cdots, m)$, and decision matrix is $Y^{\prime}=\left(y_{i j}^{\prime}\right)_{m \times n}$. 
Step 1: determine weights using the method of entropy

Evaluation indicators of freeway safety are most quantitative, for objectively reflecting the importance of indicators, the method of entropy is used to avoid the impact of subjective factors.

Efficiency and cost indicators are standardized using formula (1) and (2), standardized matrix is $Y=\left(y_{i j}\right)_{m \times n}$.

$$
\begin{gathered}
y_{i j}=y_{i j}^{\prime} / y_{\text {max }}^{\prime}(i) \\
y_{i j}=1-y_{i j}^{\prime} / y_{\text {max }}^{\prime}(i)
\end{gathered}
$$

On the basis of entropy theory, entropy of indicator $C_{i}$ is $H_{i}$, and

$$
H_{i}=-\frac{1}{\ln n} \sum_{j=1}^{n} f_{i j} \ln f_{i j}
$$

There into $f_{i j}=y_{i j} / \sum_{j=1}^{n} y_{i j}, \quad j=1,2, \cdots n$.

So, entropy weight of indicator $C_{i}$ is $w_{i}$, and

$$
w_{i}=\left(1-H_{i}\right) /\left(m-\sum_{i=1}^{m} H_{i}\right)
$$

Step 2: according to entropy weights and standardized decision matrix, weighted standardized decision matrix is $X$ :

$$
X=W Y=\left(\begin{array}{cccc}
w_{1} & 0 & \cdots & 0 \\
0 & w_{2} & \cdots & 0 \\
\vdots & \vdots & \vdots & \vdots \\
0 & \cdots & w_{j} & \cdots \\
\vdots & \vdots & \vdots & \vdots \\
0 & 0 & \cdots & w_{n}
\end{array}\right)\left(\begin{array}{cccc}
y_{11} & y_{12} & \cdots & y_{1 n} \\
y_{21} & y_{22} & \cdots & y_{2 n} \\
\vdots & \vdots & \vdots & \vdots \\
y_{i 1} & \cdots & y_{i j} & \cdots \\
\vdots & \vdots & \vdots & \vdots \\
y_{m 1} & y_{m 2} & \cdots & y_{m n}
\end{array}\right)
$$

Step 3: determine positive ideal solution $X^{+}=\left(x_{1}^{+}, x_{2}^{+}, \cdots, x_{m}^{+}\right)^{T}$ and negative ideal solution $X^{-}=\left(x_{1}^{-}, x_{2}^{-}, \cdots, x_{m}^{-}\right)^{T}$, in these formulas, and

\begin{tabular}{|c|c|c|c|c|c|c|c|c|}
\hline criterion layer & indicators & G1 & G11 & G1111 & G1211 & G10 & G1011 & G45 \\
\hline \multirow{4}{*}{ Driver $D$} & speeding $C_{11}$ (time) & 0.49 & 0.82 & 0.89 & 0.94 & 0.86 & 0.00 & 0.86 \\
\hline & $\begin{array}{l}\text { arbitrarily changing lanes } \\
C_{12} \text { (time) }\end{array}$ & 0.83 & 0.95 & 0.84 & 0.95 & 0.00 & 0.70 & 0.84 \\
\hline & $\begin{array}{c}\text { not keeping safe interval } \\
C_{13} \text { (time) }\end{array}$ & 0.46 & 0.97 & 0.98 & 1.00 & 0.00 & 0.78 & 0.97 \\
\hline & other offences $C_{14}$ (time) & 0.00 & 0.94 & 0.98 & 0.98 & 0.88 & 0.34 & 0.93 \\
\hline \multirow{3}{*}{ Vehiecle $V$} & tyre wear $C_{21}$ (time) & 0.00 & 0.92 & 0.91 & 0.96 & 0.67 & 0.27 & 1.00 \\
\hline & $\begin{array}{l}\text { bad or failing brake } \\
\qquad C_{22} \text { (time) }\end{array}$ & 0.02 & 0.82 & 0.90 & 0.90 & 0.95 & 0.00 & 0.69 \\
\hline & steering failure $C_{23}($ time$)$ & 0.00 & 0.54 & 0.60 & 0.70 & 0.36 & 0.09 & 0.06 \\
\hline \multirow{3}{*}{ Freeway $F$} & road alignment $C_{31}$ & 0.55 & 0.73 & 0.55 & 0.18 & 0.06 & 0.00 & 0.48 \\
\hline & road condition $C_{32}$ & 0.36 & 0.79 & 0.55 & 0.00 & 0.12 & 0.24 & 0.61 \\
\hline & safety facilities $C_{33}$ & 0.36 & 0.69 & 0.64 & 0.17 & 0.00 & 0.31 & 0.56 \\
\hline \multirow[t]{2}{*}{ Enviroment $E$} & $\begin{array}{c}\text { weather condition } \\
C_{41} \text { (time) }\end{array}$ & 0.00 & 0.93 & 0.96 & 0.98 & 0.20 & 0.17 & 0.95 \\
\hline & traffic flow $C_{42}$ (time/day) & 0.00 & 0.91 & 0.88 & 0.94 & 0.57 & 0.54 & 0.91 \\
\hline
\end{tabular}

$$
\begin{aligned}
& x_{i}^{+}=\max \left\{x_{i j} \mid j=1,2, \cdots, n ; \quad i=1,2, \cdots, m\right\} \\
& x_{i}^{-}=\min \left\{x_{i j} \mid j=1,2, \cdots, n ; \quad i=1,2, \cdots, m\right\}
\end{aligned}
$$

Table 2 Standardized decision matrix of evaluation indicators of Heilongjiang freeway traffic safety 
Table 3 Entropy values and entropy weights of evaluation indicators of Heilongjiang freeway traffic safety

\begin{tabular}{cccc}
\hline criterion layer & indicators & entropy values $H_{i}$ & entropy weights $w_{i}$ \\
\hline \multirow{4}{*}{ Driver $D$} & speeding $C_{11}($ time $)$ & 0.09 & 0.06 \\
& arbitrarily changing lanes $C_{12}($ time $)$ & 0.08 & 0.05 \\
& not keeping safe interval $C_{13}($ time $)$ & 0.09 & 0.06 \\
\multirow{4}{*}{ Vehiecle $V$} & other offences $C_{14}($ time $)$ & 0.10 & 0.07 \\
& tyre wear $C_{21}($ time $)$ & 0.11 & 0.07 \\
\multirow{3}{*}{ Freeway $F$} & bad or failing brake $C_{22}$ (time) & 0.17 & 0.11 \\
& steering failure $C_{23}($ time $)$ & 0.20 & 0.13 \\
\multirow{2}{*}{ Enviroment $E$} & road alignment $C_{31}$ & 0.17 & 0.11 \\
& road condition $C_{32}$ & 0.15 & 0.10 \\
& safety facilities $C_{33}$ & 0.13 & 0.08 \\
& weather condition $C_{41}$ (time) & 0.17 & 0.11 \\
\hline
\end{tabular}

Step 4: calculate the distances from positive ideal solution $S_{j}^{+}$and negative ideal solution $S_{j}^{-}$

When to calculate the distances from positive ideal solution $S_{j}^{+}$and negative ideal solution $S_{j}^{-}$, Euclidean distance is usually to be adopted.

$$
\begin{gathered}
S_{j}^{+}=\sqrt{\sum_{j=1}^{n}\left(x_{i}^{+}-x_{i j}\right)^{2}}, \quad j=1,2, \cdots n \\
S_{j}^{-}=\sqrt{\sum_{j=1}^{n}\left(x_{i}^{-}-x_{i j}\right)^{2}}, \quad j=1,2, \cdots n
\end{gathered}
$$

Step 5: calculate close degree $C_{j}^{+} \cdot C_{j}^{+}=S_{j}^{-} /\left(S_{j}^{+}+S_{j}^{-}\right)$

$C_{j}^{+}$describes the close degree of evaluation objects $R_{j}$ and positive ideal solution. The value of $C_{j}^{+}$ is bigger, $R_{j}$ is nearer to positive ideal solution, which means $R_{j}$ is safer. If $C_{j}^{+}=C_{i}^{+}$, and $S_{j}^{+}<S_{i}^{+}, x_{j}$ is better than $x_{i}$. Using formula (1) and (2), standardized decision matrix is calculated, such as in table 2. Entropy values and entropy weights are as shown in table 3.

Weighted standardized decision matrix $X$, positive ideal solution and negative ideal solution are as follow:

$$
\begin{aligned}
& X=\left[\begin{array}{lllllll}
0.0291 & 0.0492 & 0.0532 & 0.0566 & 0.0517 & 0.0000 & 0.0517 \\
0.0414 & 0.0476 & 0.0421 & 0.0474 & 0.0000 & 0.0352 & 0.0419 \\
0.0287 & 0.0580 & 0.0589 & 0.0597 & 0.0000 & 0.0466 & 0.0583 \\
0.0000 & 0.0660 & 0.0685 & 0.0687 & 0.0618 & 0.0239 & 0.0654 \\
0.0000 & 0.0645 & 0.0639 & 0.0670 & 0.0466 & 0.0190 & 0.0700 \\
0.0020 & 0.0902 & 0.0991 & 0.0991 & 0.1041 & 0.0000 & 0.0763 \\
0.0000 & 0.0704 & 0.0775 & 0.0906 & 0.0465 & 0.0119 & 0.0083 \\
0.0600 & 0.0800 & 0.0600 & 0.0200 & 0.0067 & 0.0000 & 0.0533 \\
0.0364 & 0.0788 & 0.0545 & 0.0000 & 0.0121 & 0.0242 & 0.0606 \\
0.0289 & 0.0556 & 0.0511 & 0.0133 & 0.0000 & 0.0244 & 0.0444 \\
0.0000 & 0.1024 & 0.1053 & 0.1076 & 0.0223 & 0.0186 & 0.1042 \\
0.0000 & 0.0545 & 0.0531 & 0.0563 & 0.0342 & 0.0323 & 0.0545
\end{array}\right] \\
& X^{+}=(0.0566,0.0476,0.0597,0.0687,0.0700,0.1041,0.0906,0.0800,0.0788,0.0556,0.1076,0.0563)^{T} \\
& X^{-}=(0.0000,0.0000,0.0000,0.0000,0.0000,0.0000,0.0000,0.0000,0.0000,0.0000,0.0000,0.0000)^{T}
\end{aligned}
$$


Distances from positive ideal solution $S_{j}^{+}$and negative ideal solution $S_{j}^{-}$, and rank of 7 freeways in Heilongjiang province are shown in table 4. Evaluation result shows that, the rank of these 7 national freeways of Heilongjiang province from high to low is G11, G1111, G45, G1211, G10, G1, G1011.

Table 4 Evaluation results of Heilongjiang freeway traffic safety

\begin{tabular}{cccccccc}
\hline & $\mathrm{G} 1$ & $\mathrm{G} 11$ & $\mathrm{G} 1111$ & $\mathrm{G} 1211$ & $\mathrm{G} 10$ & $\mathrm{G} 1011$ & $\mathrm{G} 45$ \\
\hline$S_{j}^{+}$ & 0.22 & 0.03 & 0.04 & 0.11 & 0.17 & 0.21 & 0.09 \\
$S_{j}^{-}$ & 0.10 & 0.24 & 0.24 & 0.23 & 0.15 & 0.08 & 0.21 \\
$C_{j}^{+}$ & 0.30 & 0.90 & 0.87 & 0.68 & 0.47 & 0.29 & 0.69 \\
rank & 6 & 1 & 2 & 4 & 5 & 7 & 3 \\
\hline
\end{tabular}

\section{References}

[1] IHT. Guidelines to Road Safety Audit. [M]. London: IHT, 1991: 12-23.

[2] ALSTROADS. Road Safety Audit (Second Edition 2002) [M]. Sydney: Standards Australia, 2002: 5-9.

[3] Wang ZX. Study on Safety Evaluation Based on Road Conditions of Freeway[D]. Changsha: Changsha University of Science \& Technology, Marster Degree Thesis, 2012: 7. (in Chinese)

[4] Li QB, Wang ZX. Research on Freeway Safety Evaluation Using Fuzzy Comprihensive Evaluation[J]. Highways \& Automotive Applications, 2012, (3): 62-65. (in Chinese)

[5] Orfila O., Coiret A., M.T. Mammar, ect. Modeling of Dynamic Vehicle-road Interactions for Safety-related Road Evaluation [J]. Accident Analysis and Prevention, 2010，42: 1736-1743.

[6] Heilongjiang traffic management bureau. Notification of road accident Heilongjiang province[R]. 2010-2014. (in Chinese) 